\title{
PERFORMANCE ANALYSIS OF DATA TRANSMISSIONS IN MPLS AND NON-MPLS NETWORKS
}

\author{
M. Asante ${ }^{1}$ and R.S. Sherratt ${ }^{2}$ \\ 1. Department of Computer science, KNUST, Kumasi, Ghana \\ 2. Signal Processing Laboratory, School of Systems Engineering, \\ The University of Reading, UK
}

\begin{abstract}
Basic Network transactions specifies that datagram from source to destination is routed through numerous routers and paths depending on the available free and uncongested paths which results in the transmission route being too long, thus incurring greater delay, jitter, congestion and reduced throughput. One of the major problems of packet switched networks is the cell delay variation or jitter. This cell delay variation is due to the queuing delay depending on the applied loading conditions. The effect of delay, jitter accumulation due to the number of nodes along transmission routes and dropped packets adds further complexity to multimedia traffic because there is no guarantee that each traffic stream will be delivered according to its own jitter constraints therefore there is the need to analyze the effects of jitter. IP routers enable a single path for the transmission of all packets. On the other hand, Multi-Protocol Label Switching (MPLS) allows separation of packet forwarding and routing characteristics to enable packets to use the appropriate routes and also optimize and control the behavior of transmission paths. Thus correcting some of the shortfalls associated with IP routing. Therefore MPLS has been utilized in the analysis for effective transmission through the various networks. This paper analyzes the effect of delay, congestion, interference, jitter and packet loss in the transmission of signals from source to destination. In effect the impact of link failures, repair paths in the various physical topologies namely bus, star, mesh and hybrid topologies are all analyzed based on standard network conditions.
\end{abstract}

Keywords: Multi-Protocol Label Switching (MPLS), Internet Protocol (IP), Delay jitter, Dropped Packet, Quality of Service (QoS)

\section{INTRODUCTION}

Rapidly growing demand for information transfer across communication networks and the need for reliable communication service have become increasingly important. It is clearly evident that businesses such as airlines, mail order, retail banking etc. can lose millions of dollars per hour in unrecoverable revenue due to a communication network failure, delay, jitter, congestion and interference from local and remote sources. The potentially drastic effects of such network impairments need to be addressed. Such shortcomings have clearly shown the need for reliable switching proce- 
dures such as the use of MPLS that provide a service which is robust to failures.

Furthermore, it has been noted in several day to day network transmissions that, as networks evolve to high bandwidth fiber optic based transmission media, the effects of even simple failures like the loss of a single link, will become more pronounced. Much of this work has been focused on the implementation of MPLS which will eliminate most of the transmission impairments and minimize the impact of component failures which results in delay, congestion and dropped packets. While there have been great strides in increasing the reliability of the physical network components, some rate of failure is inevitable. A network failure, such as the loss of a link or a node, can occur due to wide variety of reasons causing service disruptions and time delay ranging from seconds to weeks. Typical causes of network failures are accidental cable cuts, hardware malfunctions, software errors, natural disasters (e.g., floods, earthquakes, etc.), delay, jitter, congestion, dropped packets interference from local and remote links, nodes and human error (e.g., incorrect configuration of a system or maintenance etc.). Many of these causes of failures are outside the control of network providers; therefore the application of intelligent switching and routing techniques are expected to reduce network transmission impairments and also to restore the lost traffic. In this work, the use of MPLS as a means to reduce these network impairments has been proposed. Network analysis techniques utilizing MPLS has been applied within the general framework to model and analyze network performance after link failures, delay, jitter, congestion and collision. The impact of a link failure, delay and packet loss on the network performance depends on the complex interaction of several factors, some of which are the location of a failure, interference from local and remote sources within the network, the network loading, the routing algorithm, the error control procedures, and the congestion control. (Kumaki et al., 2008;Tipper et al.,1992). (Assi et al., 2003). An ingress node becoming congested will result in a knock on effect on all the intermediary nodes from the source to the destination resulting in delay, jitter and packet loss in all the nodes or links (Banerjee et al., 2001). Therefore the use of MPLS techniques has been proposed in this work as a means to reduce these impairments. Transmitted signals are normally affected by jitter which is defined as the deviation of a timing event of a signal from its intended (ideal) occurrence in time. Jitter measures the variability of delay of packets in a given stream, which is an important property for many applications (for example, streaming real-time applications). Jitter is divided into two subcomponents, Random Jitter and Deterministic Jitter.

Random Jitter is generally caused by device noise sources, e.g., thermal noise and flicker noise (Yu Chang, et al., 2008; Torkzadeh et al., 2005). The jitter increases at switches along the path of a connection due to many factors, such as conflicts with other packets wishing to use the same links and non-deterministic propagation delay in the data-link layer (Ichiyama et al., 2008; El-Henaoui et al., 1995).. The jitter accumulation at the various nodes along a transmission path has a drastic effect on the links or switches further down and will affect the QoS. Jitter control will ensure that any in -coming signal into a switch has a reduced jitter (De et al., 2009). The delay and jitter in a packet based communications network can be attributed to a number of sources. Generally speaking, the total end-to-end delay a packet experiences in a network is comprised of: the encoding delay $\left(D_{\text {enc }}\right)$ and packetization delay $\left(D_{p k t}\right)$ at the receiver, the transmission delay $\left(D_{\text {trans }}\right)$,queuing delay $\left(D_{\text {queue }}\right)$, and propagation delay $\left(P_{h}\right)$ at each hop $h$ in the path from the source to the destination, and finally the buffering delay $\left(D_{\text {buffer }}\right)$ and decoding delay $\left(D_{d e c}\right.$ at the receiver. Formally, the delay $(D)$ can be written as:

$$
D=D_{\text {enc }}+D_{p k t}+\sum\left[\left(D_{\text {trans }}+D_{\text {queue }}+P_{h}\right)+D_{\text {buffer }}+D_{\text {dec }}\right]
$$

Considering equation (1), the processing times 
such as packetization delay and transmission time will be fairly constant for all packets and it can be realized that for a given packet connection, the only significant random and changing delay component is the queuing delay $D$ quеие, thus this is the main cause of delay variation, or jitter, in such networks. In Karam et al. (2001), two sources of the variability in $D_{\text {queue }}$ are identified: variability caused by waiting behind packets from the same stream as the packet of interest, and variability caused by waiting behind packets from other locations. Queuing delay $\left(D_{\text {queue }}\right)$ needs to be controlled in order to maintain QoS in which case packets from source to destination should be monitored for delay variation from source to destination. Therefore the delay for each transaction was strictly monitored in the cause of the simulation. The analysis investigated the delay, jitter and packet loss in various network topologies as these entities can be measured in various transactions utilizing MPLS so that a packet scheduled to be carried on a resource arrives in time for its transmission. If a packet is late, or experiences excessive jitter, then it will arrive too late for transmission, and the resource scheduled for this packet will be wasted. The particular component of the communications system of interest here is the effect of the interface node (MPLS) between the fixed IP network and the source of data and the destination, the multiple access mechanism, the routers and associated entities and how this MPLS can reduce congestion, reduce delay, jitter, dropped packets and increase throughput.

Jitter compensation algorithm has been utilized to find the best compromise between jitter buffer size and packet end-to-end delay which is undesirable, especially for the delay constrained traffic expected in data transmissions. An algorithm that investigates the timing of packets to determine their level of "lateness" and delay jitter was used in the analysis. In this analysis the focus is on time-based algorithms, as they have been shown to be the most suitable for delay-constrained traffic such as that operating in a mobile communications system
(Torkzadeh et al., 2005; Vasudeva, 2009).The delay and delay variation from source to destination (jitter) is evaluated absolutely, by knowing exactly the time of transmission from the source and reception at the destination. When the exact end-to-end delay of a packet is known, the jitter can be calculated. A multimedia traffic consisting of voice, video and data transmitted along the same medium is normally affected by delay, jitter (variability in delivery times), congestion and packet loss. At this stage the impact of jitter on multimedia traffic is analyzed.

The exit time of any node ' $n$ ' from the output queue can be expressed as

$q_{n}=\left(d+r_{n}\right)$

Where ' $d$ ' is the fixed propagation delay, transmission delay, and switching delay.

$r_{n}$ is the random queuing delay of a node due to network buffers.

The node output inter-arrival time $q n$ is then given by

$q_{n}=q_{n}-q_{n-1}$

$q_{n}=\left(d+r_{n}\right)-\left[\left(d+r_{n-1}\right)-1\right]$

For a given traffic, the $\operatorname{Max}\left[q_{n}\right]$, the $\operatorname{Min}\left[q_{n}\right]$ and the Mean $\left[q_{\mathrm{n}}\right]$ values can be determined. Therefore, the jitter $J$, which represents the variation in delay at the source and the destination can be expressed as

$J=\frac{\left[\operatorname{Max}\left(\mathrm{q}_{\mathrm{n}}\right)-\operatorname{Min}\left(q_{n}\right)\right]}{\operatorname{Mean}\left(q_{n}\right)}$

This 'normalized' definition for the jitter was used in the analysis, where a Variable Bit Rate (VBR) traffic is investigated. In effect the jitter for all the transmitted signals were calculated based on equation (5). Jitter accumulation due to the increase in the number of nodes along a particular route was also analyzed for the delay, 
90

\section{Asante and Sherratt}

jitter and dropped packets. A certain observation time unit or reference time was introduced. The delay difference $(d f)$ defined as the delay at the time of leaving a source node and the delay at the time of entering a destination node was also analyzed. Now, with reference to source and destination transmissions, the source and destination with the indexes 1 and 2, respectively, their arrival times at every node input were represented by $a_{l}, a_{2}$ and their departure times by $d_{l}$ and $d_{2}$.

Applying the jitter definition as the variation in delay from source to destination, the delay difference $d f$ can be defined as;

$d f=\left(d_{2}-a_{2}\right)-\left(d_{1}-a_{1}\right)$

Where $a_{1}$ and $d_{1}$ are the arrival and departure times respectively of the source node and $a_{2}$ and $d_{2}$ are the arrival and departure times of the destination node. This ' $d f$ ' was calculated for each transmission of packets along the various links within the various topologies in which case the minimum, maximum and mean values were all determined. Traffic movement on all links in several network topologies using MPLS were examined, analyzing the delay, congestion, collisions and the throughput along the various links with and without a link failure. Mechanisms that will enable service differentiation were also implemented in which case virtual lane mapping, weighting of virtual lanes and their classification to high for voice, medium for video and low priority for data was implemented. In this case high priority traffic will pre-empt medium and medium priority will also pre-empt low priority packets in the various links in the network with and without a link failure as it was realized that a shortest path can sometimes be congested or broken which will eventually cause a delay or cause a packet to be dropped (Rahman et al., 2008; De et al., 2009). Excess traffic on a network can bring about congestion which will result in packet loss.

Therefore the introduction of a Multi-Protocol Label Switching (MPLS) based network which consists of routers and switches interconnected via transport facilities such as fiber links as illustrated in Fig.1 will go a long way to reduce packet loss in Mobile Internet Protocol (MOIP) and general network transactions. Customers connect to the backbone (core) network through Multi-Service Edge (MSE) routers (Porwal et al., 2008). The backbone comprises the core routers that provide high-speed transport and connectivity between Multi-Service Edge (MSE) routers. An MSE router contains different types of line cards and physical interfaces to provide layer 2 and layer 3 services, including Asynchronous Transmission Mode (ATM), Ethernet, IP/MPLS and Virtual Private Network (VPN) (Hodzic, and Zoric, 2008).

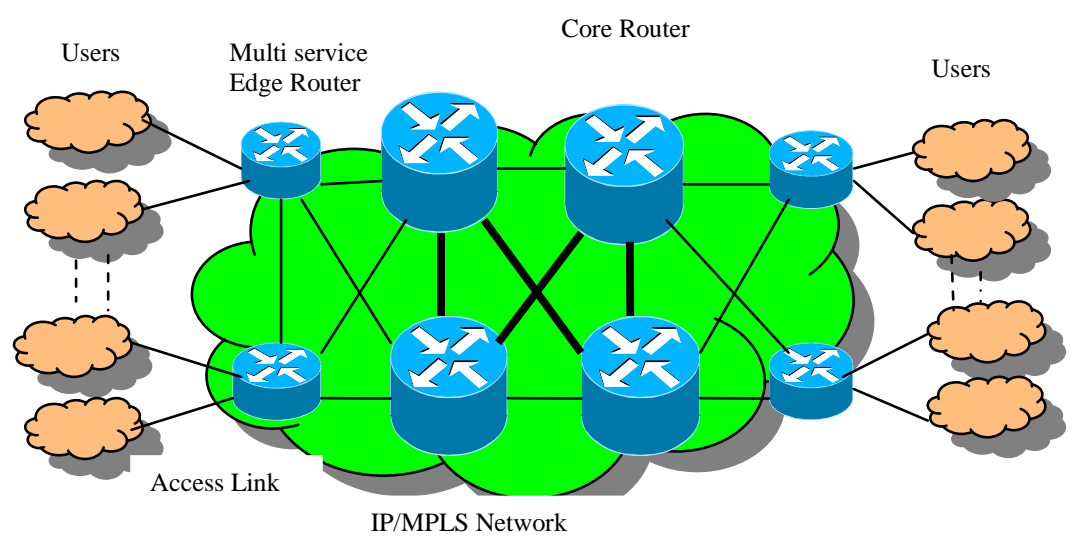

Fig. 1: Converged IP/MPLS Network Architecture (designed by authors)

Journal of Science and Technology @ KNUST December 2010 
In the incoming direction, line cards receive packets from external interfaces and forward them to the switching fabric as illustrated in Fig. 1. In the outgoing direction, line cards receive packets from the switching fabric and forward them to the outgoing interfaces (Akar et al., 2003). Switching, the heart of the router is used for switching packets between line cards. The IP/MPLS control plane which is the task performed by IP routing and MPLS signaling protocols such as Open Shortest Path First (OSPF), Intermediate System-to-Intermediate System (IS-IS) and Border Gateway Protocol (BGP) are used to advertise network topology, exchange routing information and calculate forwarding paths between routers within (intra) and between (inter) network routing domains. MPLS based traffic engineering on the other hand provides proper usage and control of the data paths ensuring a maximum use of network resources at a reduced delay, jitter, congestion, collision, interference and an increased throughput (Martini, et al., ,2009; Akar et al; 2003). Fig. 2 illustrates MPLS network with real-time application running between a media gateway and its server. From the figure, the path created along $\mathrm{ABC}$ and $\mathrm{D}$ illustrated by dashes is the shortest possible path for the transmitted packets if IP routing is used. How- ever the link between $\mathrm{B}$ and $\mathrm{C}$ can be congested due to signals from Q1 and Q2 resulting in delay, jitter, collision, interference and reduced throughput. IP routing is insensitive to congestion and will prevent a real-time application from operating effectively. The application of a MPLS-LSP will enable the transmitted signals to use alternative links (illustrated by dotted lines) or paths which will avoid the congested link B and $\mathrm{C}$ within the network. In the event of any link or node failure along the dashed route and the dotted routes, the transmitted signal can use the primary link as an alternative path (Rahman et al., 2008).

The internet is perceived as a giant and complex network which comprises of several networks such as LANs, WANs and MANs linked together by either wire or wireless in which case the nodes, paths/links experience variable transmission impairments resulting in reduced throughput. Therefore a hybrid network illustrated in Fig.3 was used in the analysis so as to expose the packets to the variable conditions experienced by transmitted signals in the internet. MPLS was introduced at the ingress and the egress nodes in all the networks. In this experiment, the delay, jitter accumulation and dropped packets were all recorded.

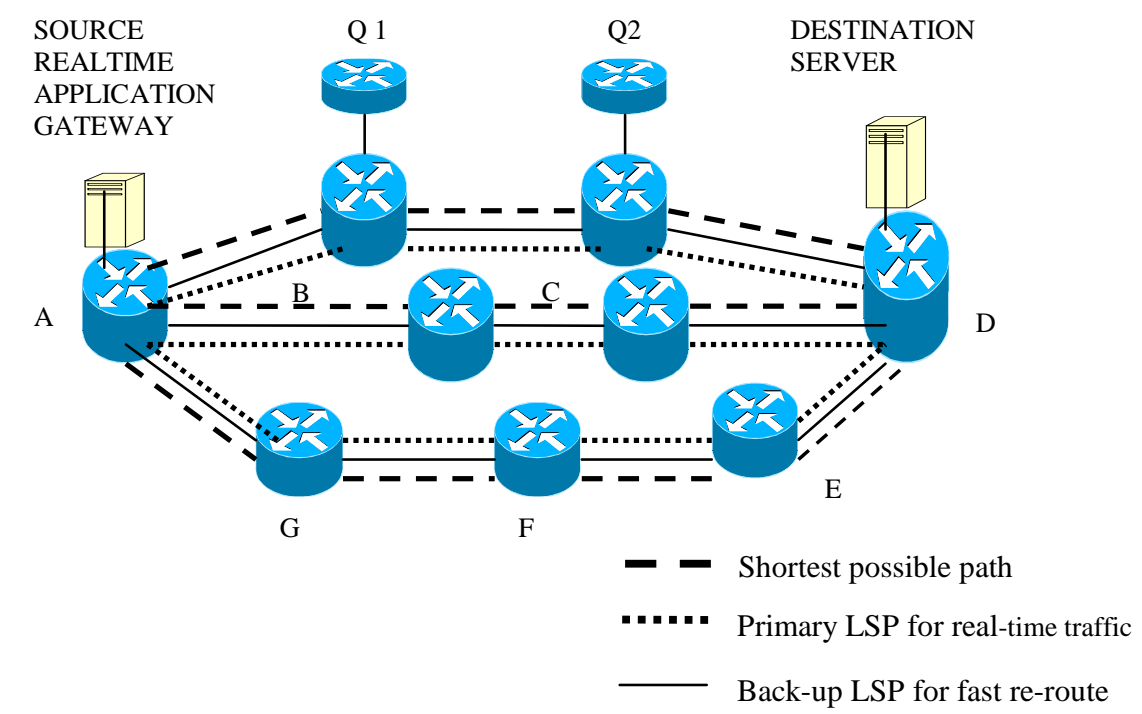

Fig. 2: MPLS Network in a Real-Time Application (designed by authors)

Journal of Science and Technology ㄷ KNUST December 2010 


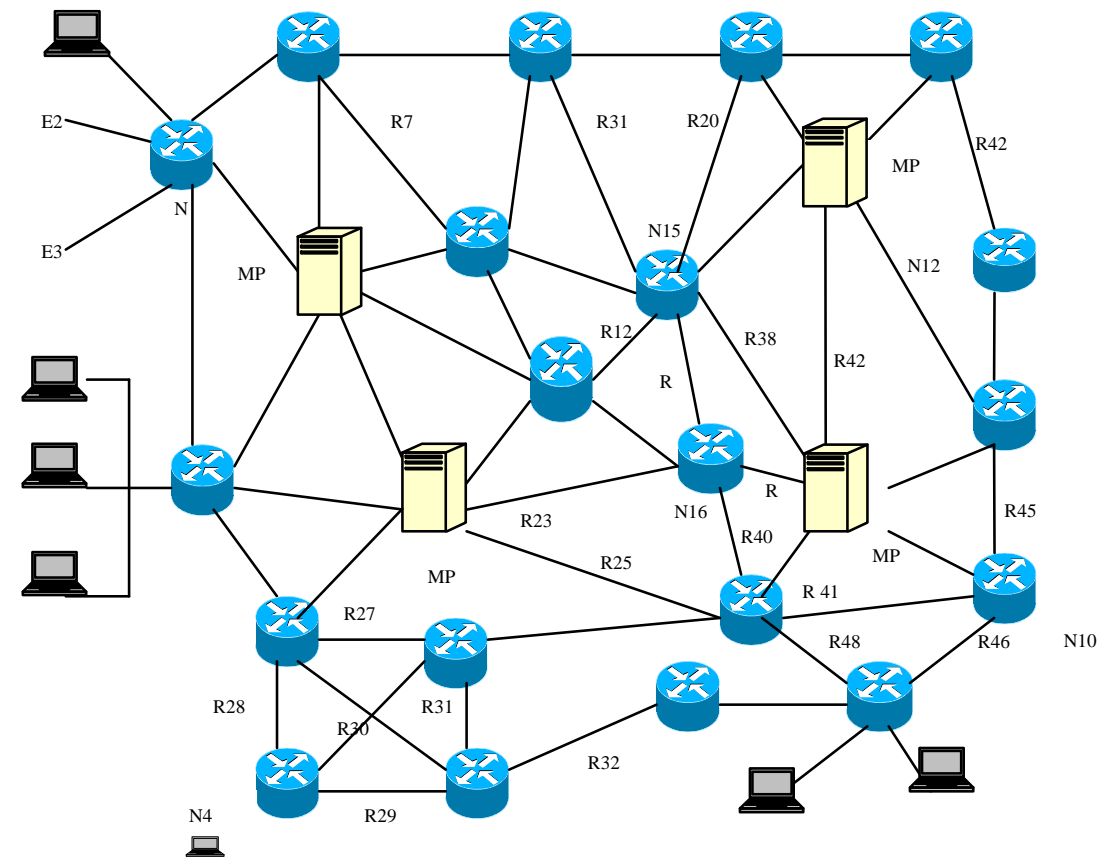

Fig. 3: Hybrid Mesh Topology (designed by authors)

\section{METHODOLOGY}

To monitor the performance of MPLS and the effect of delay, jitter, congestion and interference from remote sources a test bed illustrated in Fig. 2, 3 and the normal network topologies (bus, star, ring, mesh) were built in turn and a series of simulations were performed and analyzed on a network abstraction software simulator; Prophecy, thereby calculating the jitter using equation (5) in each case on transmitted signals along the various transmission paths within the various topologies and modifying the topology as was required. In this scenario, data from various edge nodes were directed at the ingress nodes or sources within the various transmission routes in the various topologies illustrated in Fig. 2 and 3 to the various egress nodes for onward transmission to various destinations in turn. The delay, jitter, transmitted and dropped packets were measured based on; single-repair path and non-prioritized, singlerepair path and prioritized, double-repair path and non-prioritized, double-repair path and prioritized, multi-repair path and non priori- tized, multi-repair path and prioritized. The time and the size of packets were specified at the beginning of every run of the simulation. Packet sizes of $(100 \ldots 1000,2000$, $3000 \ldots . .5000 \mathrm{~K})$ were transmitted through the various links in the various networks topologies in turn. Each simulation was run 15 times in which case the mean values for delay, transmitted and dropped packets for all the fifteen simulations were taken as the representative value. In the course of the simulation, packets from other nodes were introduced into the network to test for the behavior of the various nodes and links with respect to interference, congestion, delay and jitter from local and remote signals and how the action of the MPLS will react to the traffic transmissions under those conditions. Bandwidth size of $2 \mathrm{Mb}$ was chosen for all the runs. In the course of the simulation, some links were severed based on single, double, and multi -link failure and after the run of several simulations the delay, jitter, transmitted packets and the number of dropped packets were also recorded. The packets were further prioritized 
under high for voice, medium for video and low for data and the delay, jitter, and dropped packets were all measured in each case.

The following assumptions were made in the course of the simulation.

1. The bandwidth was assumed to be the same along all transmission paths.

2. Transmission along a failed link was ex pected to be re-routed to other paths that were determined using the shortest possi ble path approach.

3. Transmission delay between a source and a destination pair was measured by the dif ference in transmission times between the source and destination.

4. The link distance between any two nodes was assumed to be equal.

5. The average rate of traffic flow from each node to another node was based on the link or node environment.

\section{Multiplexing Strategies}

The delay and jitter parameters were investigated under two different multiplexing schemes. The first scheme multiplexes the traffic in the same Virtual Path (VP), "All in one VP", while the other multiplexes each traffic component in a separate VP, "One VP for each" that is all the signals were prioritized, High for Voice, Medium for Video and Low, for Data. In effect each path was divided into three virtual paths. It is known from the resource allocation point of view that the one VP for each type of signal is preferable, since it provides the appropriate quality-of-service requirements for each traffic components. By this analysis, the impact of the delay, jitter, and dropped packets on the transmitted signals were determined based on single, double and multirepair paths which are non-prioritized and with and without repair paths and prioritized with and without repair paths. The main aim of the analysis was to analyze delay/transmission flow, jitter/transmission flow and jitter/dropped packets per each transmission in the various links within the various topologies as a way of reducing these impairments using MPLS. These simple topologies were chosen so that packets in flight can be observed and the use of the hybrid mesh topology in Fig. 3 was to create the environment experienced by transmitted signals on the internet which is a giant network of different topologies combined together (hybrid network). The simulation was performed based on the following parameters:

Packet size: $100 \mathrm{~K}, 200 \mathrm{~K}$ up to $5000 \mathrm{~K}$,

Transmission speed: 2 Mbps

Simulation duration : $5 \mathrm{~s}$

Group jitter value mean : $0.3 \mathrm{~s}$

Individual packet jitter variance from mean: $0.001 \mathrm{~s}$

\section{RESULTS AND DISCUSSION}

The delay, transmitted packets, jitter and dropped packets were similar in all the simulation runs and were also proportional to the various sizes of packets transmitted and dropped packets were also proportional to the sizes of packets transmitted. From simulations conducted on the bus topology, star topology, mesh topology and the hybrid network, it was observed that the delay, jitter, dropped and interference increased in the absence of a MPLS and non-prioritized routes and the presence of MPLS and prioritized routes reduced the transmission impairments drastically.

\section{Single-repair Paths}

Figure 4 presents a graph of transmitted packets against delay along the routes, R1-R2-R28R30, R4-R8-R25-R47，R5-R6-R30-R13-R37R18， R5-R15-R16-R17-R42-R44， R31-R21R40-R48 and R44-R49-R39-R24 in figure 3 along a single repair path, non-prioritized and using MPLS. It can be inferred from the graph that the delay increased greatly with a corresponding decrease in the number of transmitted packets along all the routes. This shows that the absence of prioritized signals along a single repair path led to the packets experiencing higher delay and a decrease in the number of transmitted signals. In effect signals were subjected to higher delay, collision, congestion and interference. 


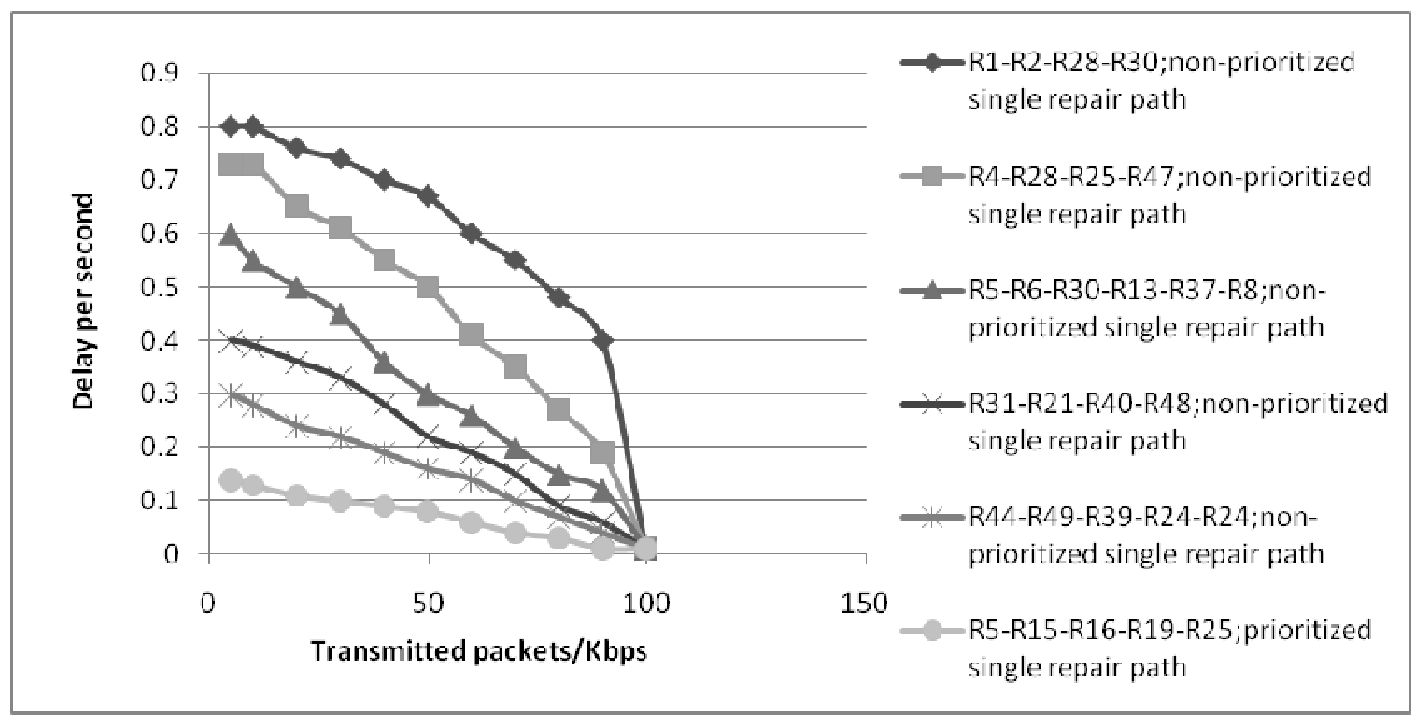

Fig4: Delay versus Transmitted packets in a Hybrid-Mesh network, non- prioritized in a single repair path within an MPLS environment

Figure 5 presents a graph of transmitted packets against jitter on a single repair path and nonprioritized paths along the routes R1-R2-R28R30, R4-R8-R25-R47, R5-R6-R30-R13-R37R18, R5-R15-R16-R17-R42-R44， R31-R21R40-R48 and R44-R49-R39-R24 in Fig.3. It can be inferred from the figure that the number of transmitted packets decreased significantly with a corresponding increase in jitter along the various routes. This indicates that packets transmitted under such conditions experienced a greater delay, congestion, collision and interference causing a lot of packets to be dropped.

\section{Double-repair Paths}

Figure 6 presents a graph of transmitted packets against delay and it illustrates the effect of creating double repair paths in the various transmission paths namely, R1-R2-R28-R30, R4-R8 -R25-R47, R5-R6-R30-R13-R37-R18, R5-R15R16-R17-R42-R44， R31-R21-R40-R48 and R44-R49-R39-R14 in Fig.3. From Fig.6, it can be inferred that the delay increased significantly with a corresponding decrease in the number of transmitted packets along non- prioritized double repair paths. This is due to the fact that a lot more packets were dropped due to the greater transmission impairments experienced during transmission. However using a non-prioritized double repair path reduced the delay to a greater extent and more packets were transmitted at reduced delay than in the use of non-prioritized single repair path in Fig.5.This shows that the creation of a double repair path has an effect on the transmitted signals.

Figure 7 presents a graph of transmitted packets against jitter in a non-prioritized double repair path along routes R1-R2-R28-R30, R4-R8-R25 -R47, R5-R6-R30-R13-R37-R18, R5-R15-R16R17-R42-R44, R31-R21-R40-R48 and R44R49-R39-R24 in Fig.3. From the figure it can be inferred that the jitter was lower and more packets were transmitted compared to the use of single repair path in Fig. 5 even though MPLS was utilized in each case. This shows that the creation of the double repair path increased the rate of transmission significantly reducing the transmission impairments. 


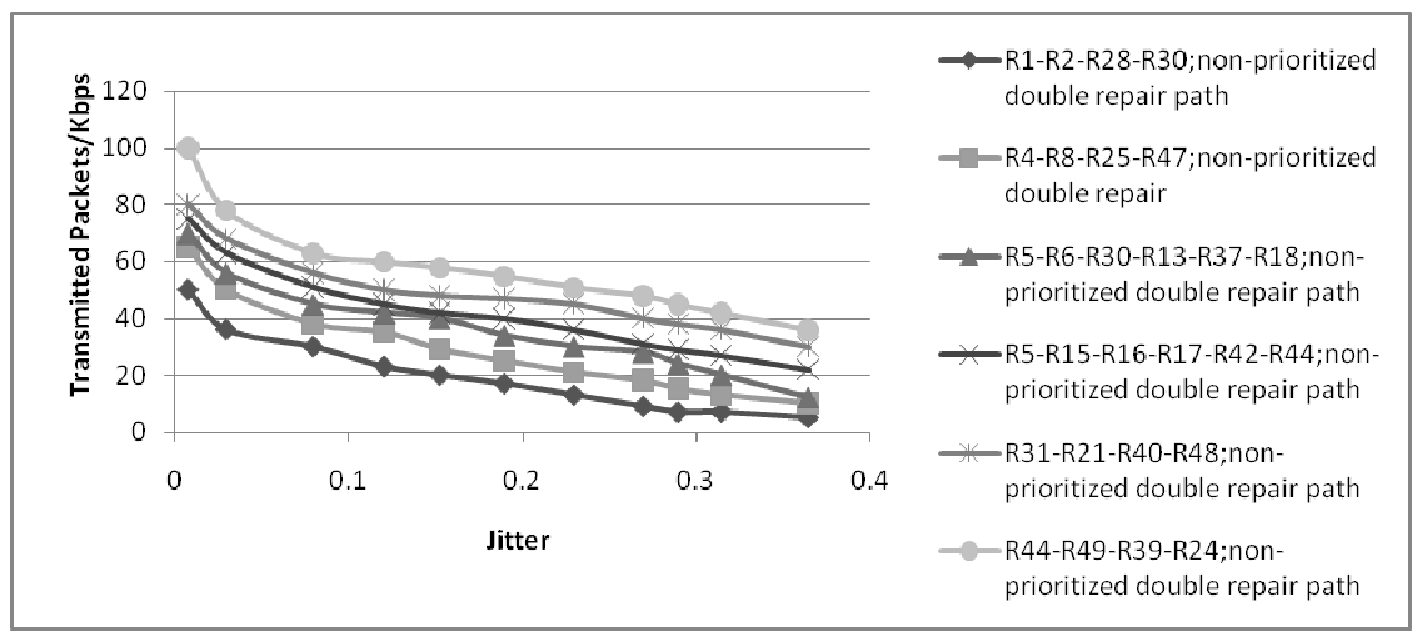

Fig 5: Transmitted packets versus Jitter in a Hybrid-Mesh network, non-prioritized in a single repair path within an MPLS environment

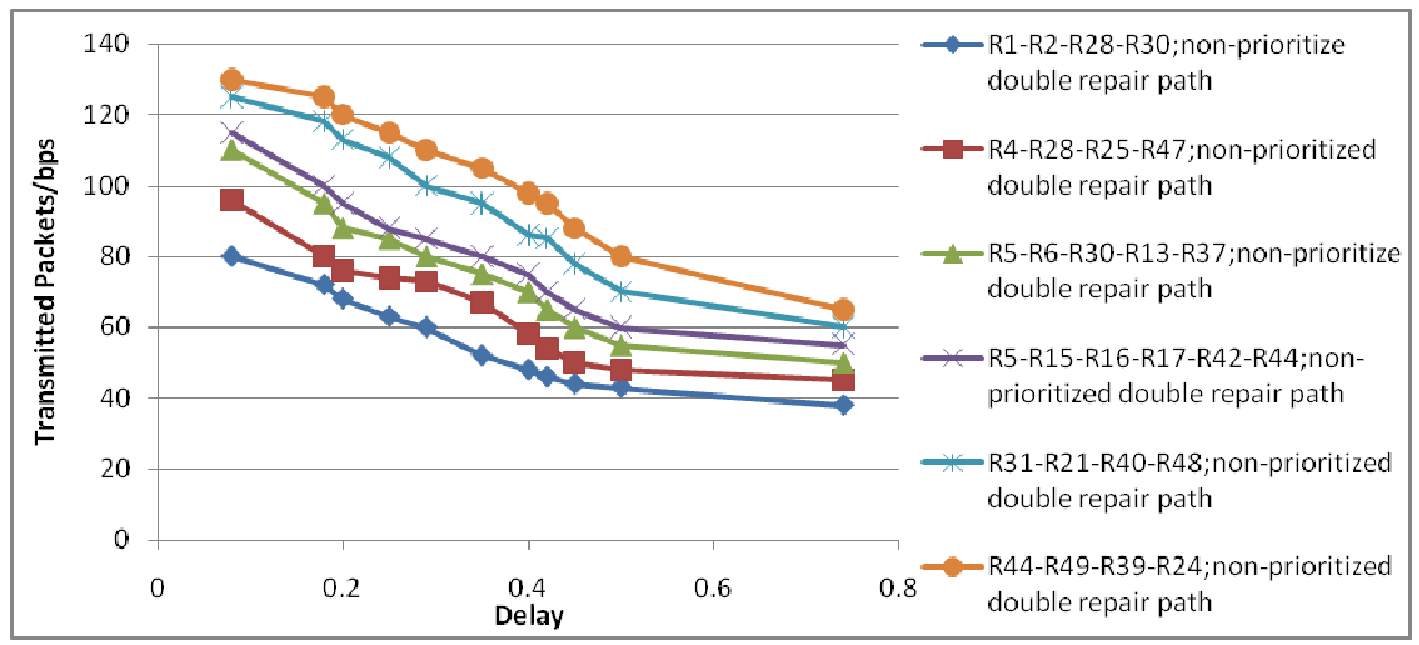

Fig. 6: Delay versus Transmitted packets in a non-prioritized double-repair paths within an MPLS environment

Figure 8 presents a graph of delay against transmitted packets in a prioritized double-repair path along the paths R1-R2-R28-R30, R4-R8R25-R47, R5-R6-R30-R13-R37-R18, R5-R15R16-R17-R42-R44, R31-R21-R40-R48 and R44-R49-R39-R24 in Fig.3. It shows that the delay decreases at a corresponding increase in transmitted packets as compared to Fig.7 in which case the delay is less and the number of transmitted packets are greater than in the case of Fig.5 where the transmission paths were double-repair and non-prioritized. Therefore the presence of the double-repair path and the prioritization of the signals in Fig.8 led to a reduction in the delay and the number of packets transmitted increased accordingly. 


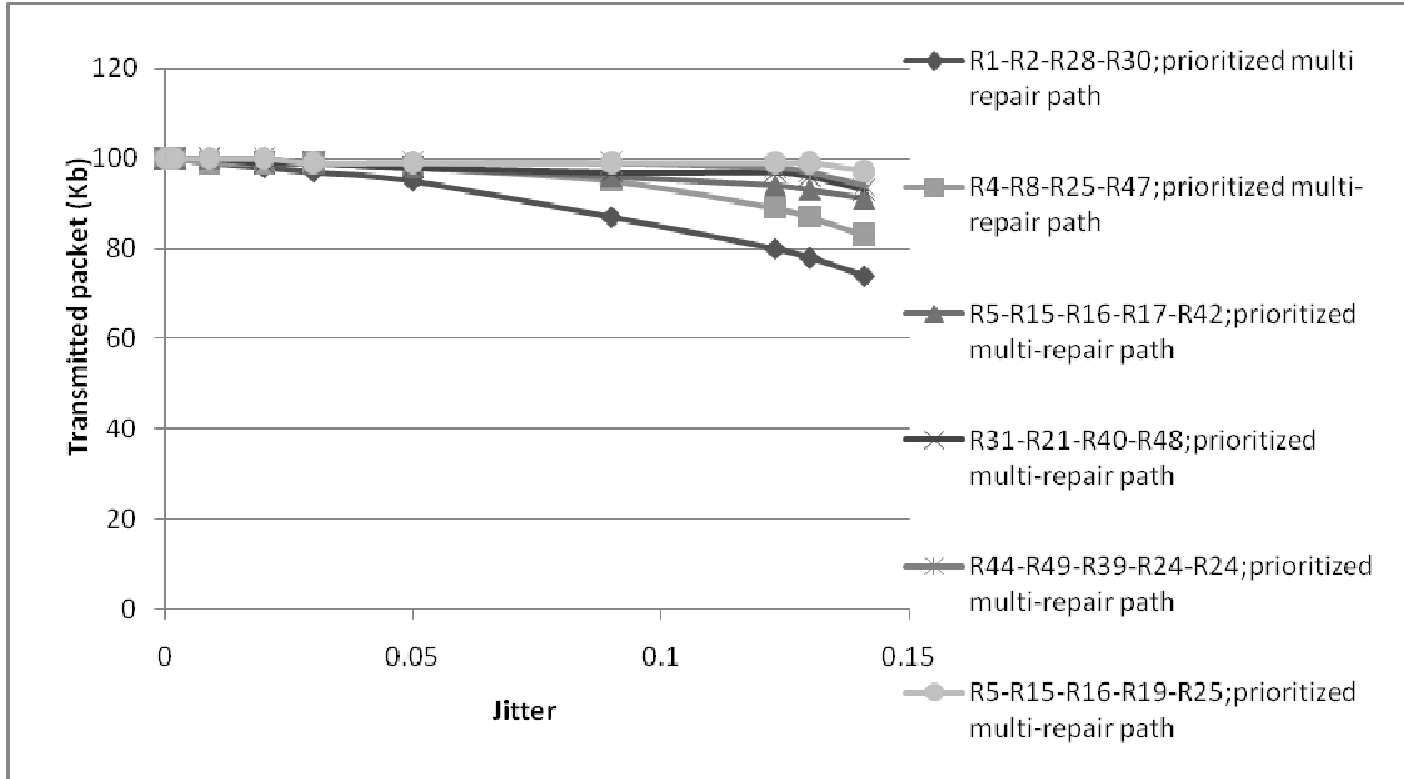

Fig.7: Transmitted versus Jitter in Hybrid-Mesh network, non-prioritized in Double- repair paths within an MPLS environment

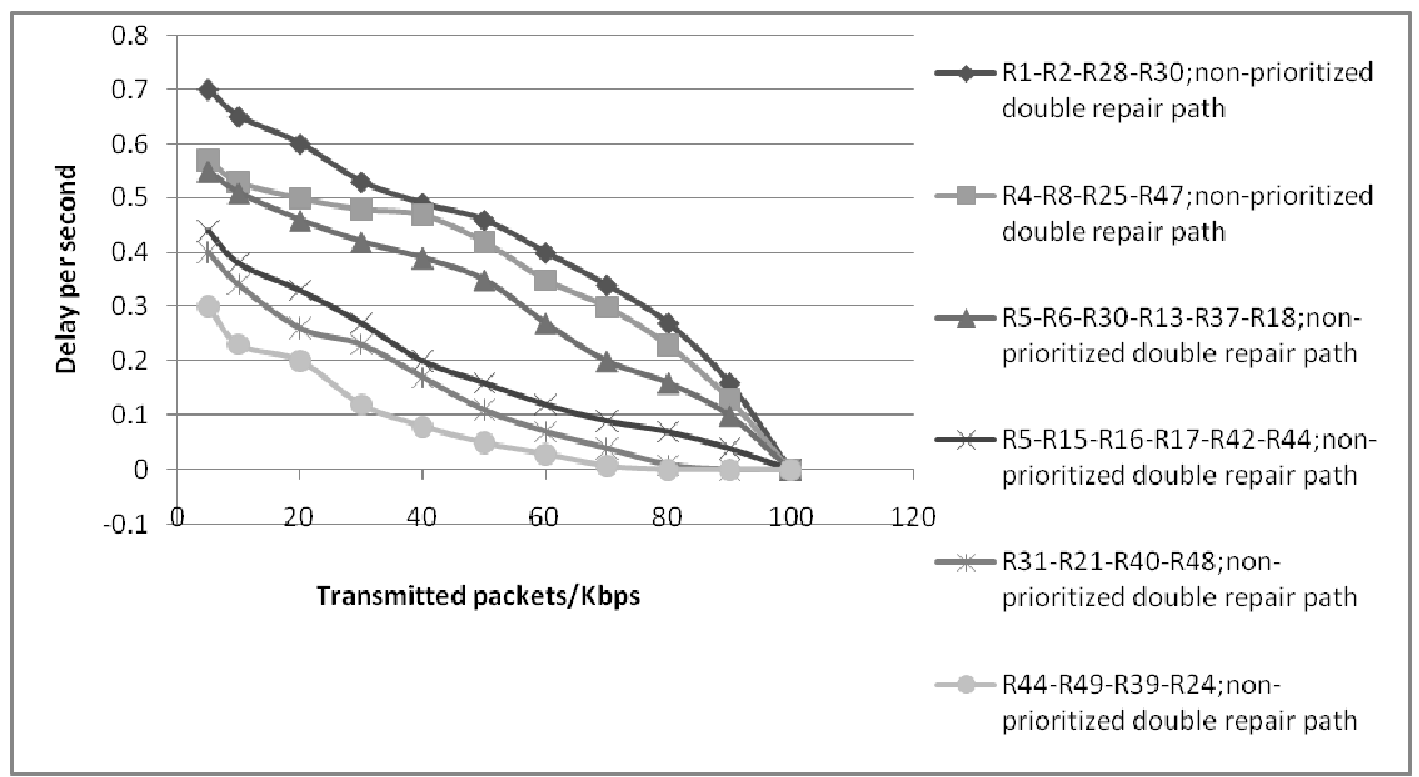

Figure 8: Delay versus Transmitted packets in hybrid-Mesh network, prioritized in doublerepair paths within an MPLS environment 
Figure 9 presents a graph transmitted packets against jitter in a prioritized double-repair path within an MPLS environment along the routes R1-R2-R28-R30, R4-R8-R25-R47, R5-R6-R30 -R13-R37-R18,R5-R15-R16-R17-R42-R44, R31-R21-R40-R48 and R44-R49-R39-R24 in fig3. From the graph it can be inferred that prioritizing the signals in a double-repair path reduces the jitter and increases the number of transmitted packets. Compared to the nonprioritized double-repair path in Fig.7, the prioritized double-repair path transmits signals at a reduced delay, jitter, congestion, collision interference and increased throughput.

\section{Multi-repair Paths}

Figure 10 presents a graph of delay against transmitted signals within a non-prioritized multi-repair path along the paths R1-R2-R28R30，R4-R8-R25-R47，R5-R6-R30-R13-R37R18, R5-R15-R16-R17-R42-R44， R31-R21R40-R48 and R44-R49-R39-R24 in Fig.3. It can be inferred from the graph that more packets were transmitted and the delay in each case was less compared to the situation in both nonprioritized single and double repair-paths. In which case the effect of delay, congestion, jitter, collision and interference were all reduced to a greater extent and performance was better than the utilization of both non-prioritized single and double-repair paths.

Figure11 presents a graph of transmitted packets against jitter along the routes R1-R2-R28R30，R4-R8-R25-R47，R5-R6-R30-R13-R37R18， R5-R15-R16-R17-R42-R44， R31-R21R40-R48 and R44-R49-R39-R24 in Fig.3 in a non-prioritized multi-repair path. From the graph it can inferred that the creation of a multi -repair path reduced the jitter and this led to an increase in the number of transmitted packets along the various routes. The investigation revealed that an unknown number of remote nodes may interfere with signals towards the ingress/egress LSP or node especially when the medium of transmission is wireless. In this situation a dimensionally/topologically shortest path ceases to be a shortest path as a dimensionally/topologically longer path will sometimes experienced less delay, less congestion and less collision making it more efficient than the dimensionally shortest path with higher

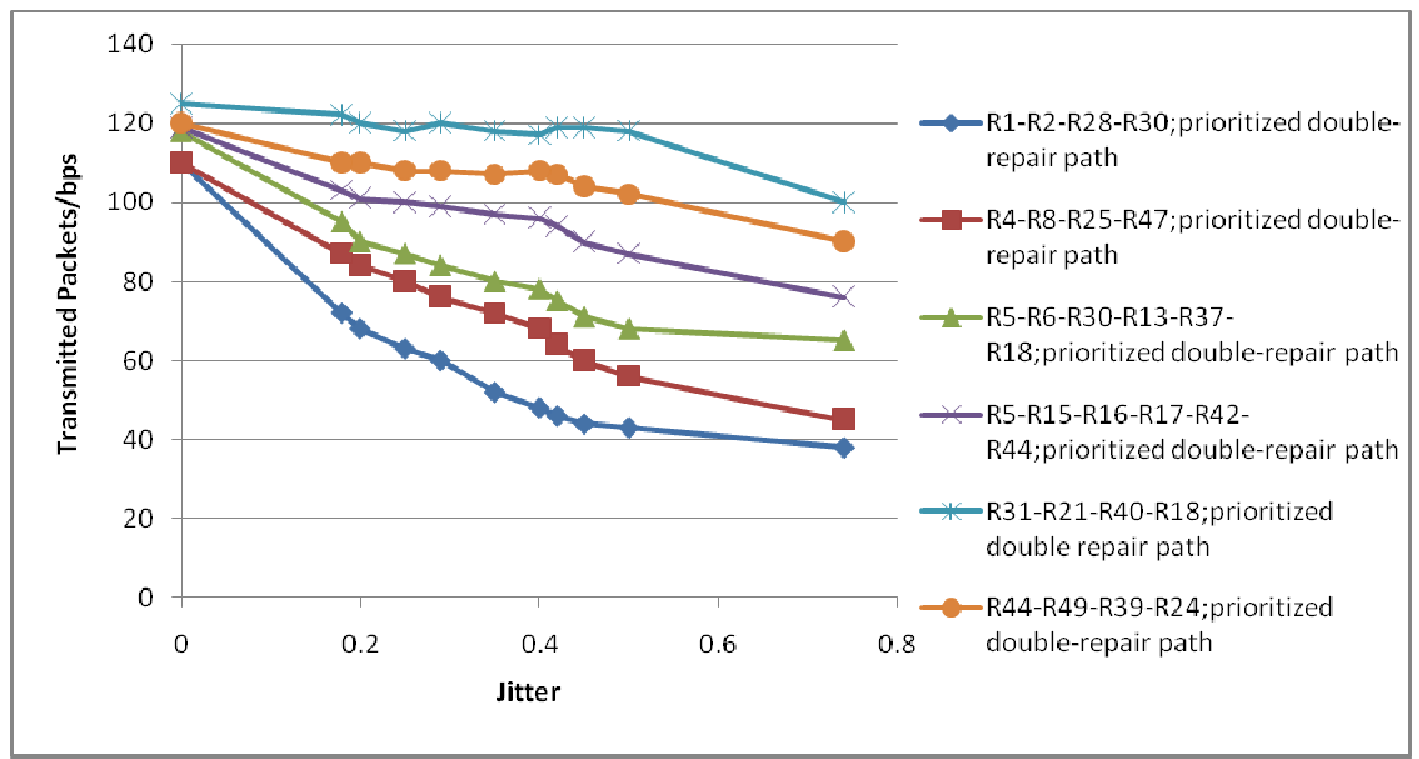

Fig. 9: Transmitted packets versus Jitter in a hybrid-Mesh network, prioritized in doublerepair paths within an MPLS environment 


\section{Asante and Sherratt}

delay, congestion and higher collision with a corresponding fewer transmitted packets. Generally it was observed that the type of multiplexing scheme applied is quite significant for the delay, jitter values and the number of dropped packets. However, prioritizing the signals and the creation of multi-repair paths highly influenced the delay, jitter and the number of dropped packets within a transmission. The worst degradation appears when there are no repair paths and no prioritization. It was also observed that as the number of nodes increases along a particular path, the delay, jitter and the number of dropped packets increases with a corresponding decrease in the transmitted packets. Again the difference in delay between the shorter and longer paths was minimal because several signals both internal and remote were using the shorter paths other than the longer paths and this rendered it more congested and an increase in delay, collision and a decreased throughput. In any case the longer paths experienced less delay congestion and collision because most of the signals tried to avoid them. Furthermore, it was found out that the delays after prioritizing the signals into high for voice, medium for video and low for data in the pres- ence of a single, double and multiple links with and without MPLS were very low.

\section{CONCLUSION}

Signal transmissions in various transmission mediums along different transmission links are normally affected by the nature of the transmission medium, the state of the transmission path, the number of nodes along a particular path and the effect of local and remote signals transmitting to or away from a particular path or node. From the simulations conducted, it has been established that the creation of prioritized single, double and multi-repair paths in MPLS environment reduces the effect of transmission impairments such as delay, jitter, collision, congestion, and interference and that prioritized multi- repair paths produces a greater throughput than double-repair paths and double-repair paths in turn is also more effective than single repair paths. Again the utilization of repairpaths whenever there was a failure has also led to a decrease in the effect of delay, jitter, congestion and interference. Therefore the analysis which seeks to eliminate the transmission impairments such as delay, congestion, and jitter has been achieved using prioritized single, prio-

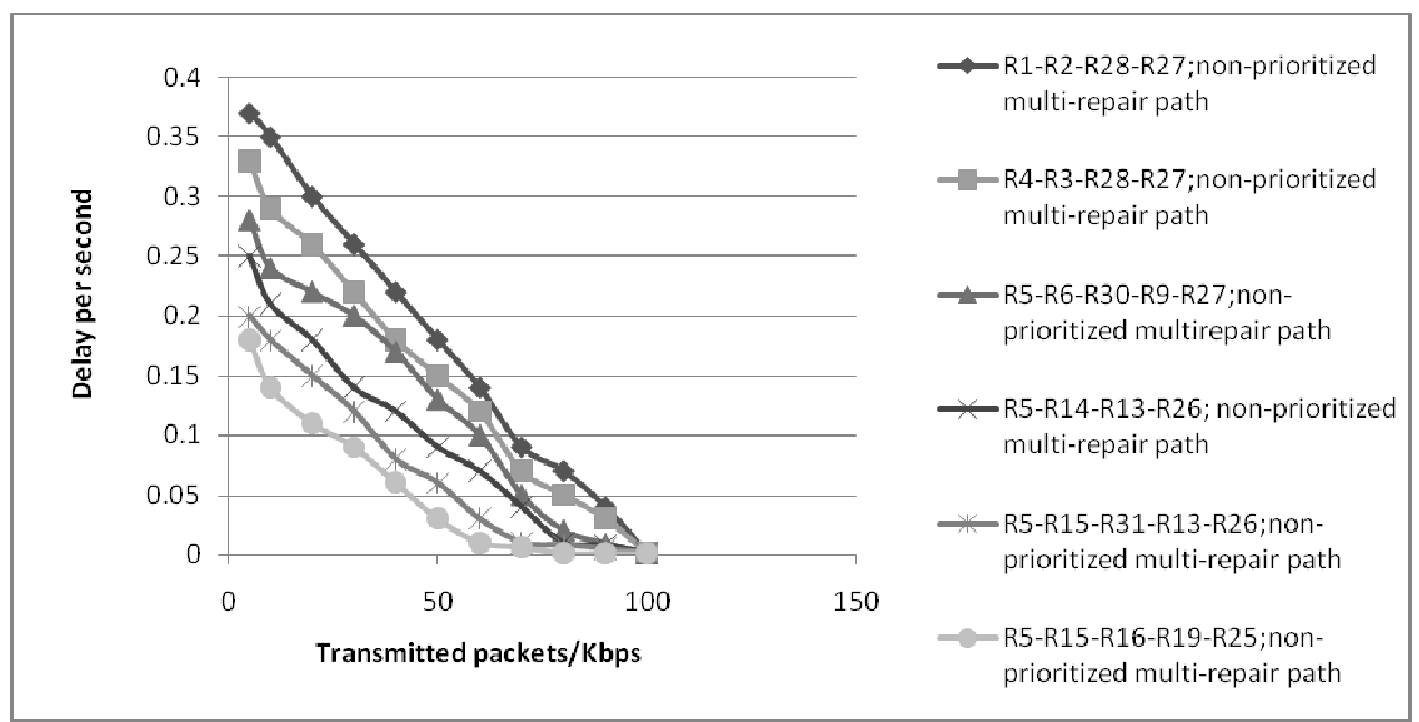

Fig. 10: Delay versus Transmitted packets in a hybrid-Mesh network, non-prioritized in multi-repair paths within an MPLS environment 
Data transmissions in MPLS and non-MPLS networks

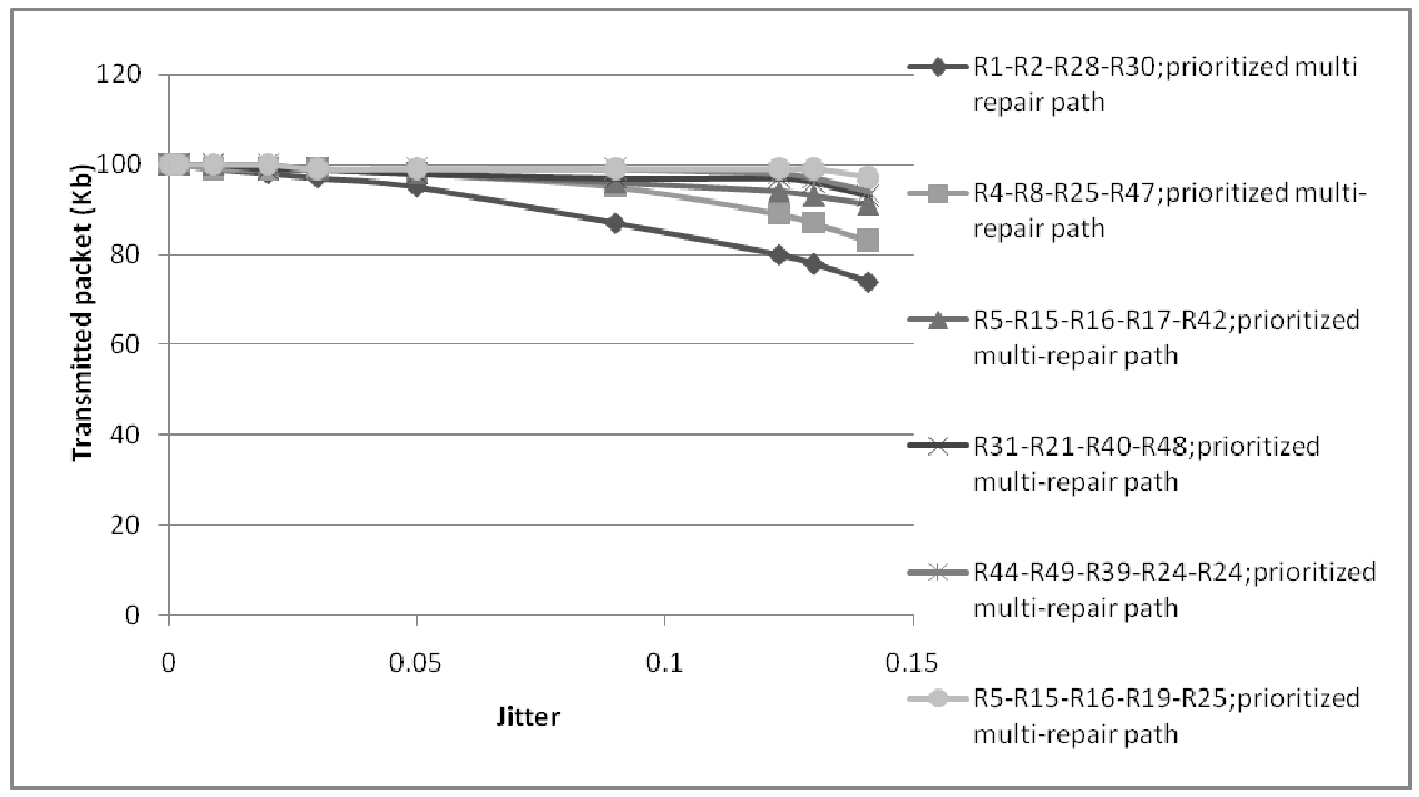

Figure 11: Transmitted packets versus Jitter in a hybrid-Mesh network, prioritized in multirepair paths within an MPLS environment

ritized double and prioritized multi-repair paths all in an MPLS environment. It has also been demonstrated that there has been a reduction in delay, jitter, congestion, collision and interference hence the longer path which should have been traversed by transmitted packets from source to destination in normal routing procedures in the absence of MPLS has been reduced.

\section{REFERENCES}

Akar, N., Atik, M., and Karasan, E. (2003). A Re-ordering-free Multi-path Traffic Engineering Architecture for Diffserv MPLS Networks. IEEE 3rd Workshop on IP Operations and Management, 107-113.

Assi, C. M., Yinghua Ye., Sudhir Dixit and Ali, M.A (2003). Dynamic bandwidth allocation for quality-of-service over Ethernet PONs, IEEE Journal on Selected Areas in Communications, 21(9), 1467 1477.

Banerjee, A., Drake, J., Lang, J., Turner, B.,
Awduche, D., Berger, L., Komella, K and Movaz, Y (2001). An overview of signaling enhancements and recovery techniques" IEEE Communication Magazine, 39(7): 144- 151

De, P. Mann, V and Mittaly, U (2009). Handling OS jitter on multicore multithreaded Systems, IEEE International Symposium on Parallel and Distributed Processing 1 12

El-Henaoui, S., Coelho, R., Tohme, S (1995). Inter-flows jitter analysis for multimedia traffic in ATM networks. Computers and Communications, Proceedings of IEEE Symposium, 377-383

Hodzic, $\mathrm{H}$ and Zoric, S (2008). Traffic engineering with constraint based routing in MPLS Networks, $50^{\text {th }}$ International Symposium, ELMAR, 2008, 1:269 - 272

Ichiyama, K., Ishida, M., Yamaguchi, T. J and Soma, M (2008). Novel CMOS Circuits to 


\section{Asante and Sherratt}

Measure Data- Dependent Jitter, Random Jitter, and Sinusoidal Jitter in Real Time. IEEE Transactions on Microwave Techniques and transactions 56, Issue5 Part 2, $1278-1285$

Karam, M. J and Tobagi, F. A. (2001). Analysis of the delay and jitter of voice traffic over the Internet, Proceedings of the Twentieth Annual Joint Conference of IEEE and Computer Societies on of a P2MP MPLS-based hierarchical service management System. 794 - 799, IEEE Symposium on Computers and Communications $2: 824-833$

Martini, B., Baroncelli, F., Martini, V., Torkman K. and Castoldi, P. (2009) ITU-T RACF implementation for applicationdriven QoS control in MPLS networks. IFIP/IEEE International symposium on Integrated network management, 422 429

Porwal, M. K., Yadav, A and Charhate, S. V. (2008). Traffic Analysis of MPLS and Non MPLS Network including MPLS Signaling Protocols and Traffic Distribution in OSPF and MPLS. First International conference on Emerging trends and Technology, $187-192$
Rahman, M. A., Kabir, A. H., Lutfullah, K. A. M.,Hassan, M. Z and Amin,M. R (2008). Performance analysis and the study of the behavior of MPLS protocols International conference on Communication Engineering, $226-229$

Tipper, D., Hammond, J., Balakrishnan, K., Menon, S., Sharma, S., Khetan, and A., Hang, W. (1992). A study of network recovery from link and node failures with emphasis on transient behavior of routing algorithms. Tech Rep. ECE Dept., Univ. of Clemson, SC

Torkzadeh, P., Tajalli, A and Mojtaba (2005). Analysis of jitter peaking and jitter accumulation in re-circulating delay-locked loops. IEEE International Symposium on Circuits and Systems, ISCAS 2005(3): $2255-2258$

Vasudevan, V. (2009) Analysis of Clock Jitter in Continuous-Time Sigma-Delta Modulators. IEEE Transactions on Circuits and Systems: Regular Papers, 56, 519 - 528

Yu Chang., Dan Oh and Madden, C. (2008). Jitter modeling in statistical link Simulation, IEEE Symposium on Electronic compatibility, 1 - 4 